\title{
Exceptional parallelisms characterize the evolutionary transition to live birth in phrynosomatid lizards
}

Saúl Domínguez-Guerrero ( $\sim$ sauldguerrero@gmail.com )

Yale University https://orcid.org/0000-0001-5323-6752

Fausto Méndez-de la Cruz

Universidad Nacional Autónoma de México

Norma Manríquez-Morán

Universidad Autónoma del Estado de Hidalgo

Mark Olson

National Autonomous University of Mexico https://orcid.org/0000-0003-3715-4567

\section{Patricia Galina-Tessaro}

Centro de Investigaciones Biológicas del Noroeste S. C.

\section{Diego Arenas-Moreno}

Universidad Nacional Autónoma de México

Adán Bautista-del Moral

Universidad Nacional Autónoma de México

Adriana Benítez-Villaseñor

Universidad Nacional Autónoma de México

Héctor Gadsden

Instituto de Ecología A. C. https://orcid.org/0000-0002-1164-0578

Rafael Lara-Reséndiz

Universidad Nacional de Córdoba

Carlos Maciel-Mata

Universidad Autónoma del Estado de Hidalgo

Francisco Muñoz-Nolasco

Universidad Nacional Autónoma de México

Rufino Santos-Bibiano

Universidad Nacional Autónoma de México

Jorge Valdez-Villavicencio

Conservación de Fauna del Noroeste, A. C.

Guillermo Woolrich-Piña

Tecnológico Nacional de México campus Zacapoaxtla

Martha Muñoz 


\section{Article}

Keywords: viviparity, viviparous lizards, evolutionary transition, live birth

Posted Date: January 12th, 2021

DOl: https://doi.org/10.21203/rs.3.rs-127587/v1

License: (c) (i) This work is licensed under a Creative Commons Attribution 4.0 International License. Read Full License

Version of Record: A version of this preprint was published at Nature Communications on May 24th, 2022. See the published version at https://doi.org/10.1038/s41467-022-30535-w. 


\section{EXCEPTIONAL PARALLELISMS CHARACTERIZE THE EVOLUTIONARY TRANSITION TO LIVE BIRTH IN PHRYNOSOMATID LIZARDS}

Saúl F. Domínguez-Guerrero ${ }^{1,2,3^{*}}$, Fausto R. Méndez-de la $\mathrm{Cruz}^{2}$, Norma L. ManríquezMorán $^{4}$, Mark E. Olson ${ }^{2}$, Patricia Galina-Tessaro ${ }^{5}$, Diego M. Arenas-Moreno ${ }^{2,3}$, Adán Bautista-del Moral², Adriana Benítez-Villaseñor ${ }^{2,3}$, Héctor Gadsden ${ }^{6}$, Rafael A. LaraReséndiz $^{5,7}$, Carlos A. Maciel-Mata ${ }^{4}$, Francisco J. Muñoz-Nolasco ${ }^{2,3}$, Rufino SantosBibiano $^{2,3}$, Jorge H. Valdez-Villavicencio ${ }^{8}$, Guillermo A. Woolrich-Piña ${ }^{9}$, and Martha M. Muñoz ${ }^{1}$

${ }^{1}$ Department of Ecology and Evolutionary Biology, Yale University, 06511 New Haven CT, USA. ${ }^{2}$ Instituto de Biología, Universidad Nacional Autónoma de México, 04510 Ciudad de México, México. ${ }^{3}$ Posgrado en Ciencias Biológicas, Instituto de Biología, Universidad Nacional Autónoma de México, 04510 Ciudad de México, México. ${ }^{4}$ Centro de Investigaciones Biológicas, Universidad Autónoma del Estado de Hidalgo, 42184 Mineral de la Reforma, Hidalgo, México. ${ }^{5}$ Centro de Investigaciones Biológicas del Noroeste S. C., 23096 La Paz, Baja California Sur, México. ${ }^{6}$ Instituto de Ecología, A. C., 61600 Pátzcuaro, Michoacán, México. ${ }^{7}$ Facultad de Ciencias Exactas, Físicas y Naturales, Centro de Zoología Aplicada and Consejo Nacional de Investigaciones Científicas y Técnicas, Instituto de Diversidad y Ecología Animal, Universidad Nacional de Córdoba, 5000 Córdoba, Argentina. ${ }^{8}$ Conservación de Fauna del Noroeste, A.C., 22785 Ensenada, Baja California, México. ${ }^{9}$ Tecnológico Nacional de México campus Zacapoaxtla, 73680 Zacapoaxtla, Puebla, México.

*e-mail: sauldguerrero@gmail.com 


\begin{abstract}
Viviparity is an evolutionary innovation that enhances maternal protection of developing embryos relative to egg-laying ancestors. The behavioral, physiological, morphological, and life history pathways underpinning this innovation, however, remain unclear. We capitalized on the repeated origin of viviparity in phrynosomatid lizards to tease apart the phenotypic patterns associated with evolutionary transitions to live birth. We detected tandem reductions in mass-specific metabolic rate and mass-specific production in viviparous lineages, in turn reflecting decreases in thermal physiology and fecundity, respectively. These pathways reduce the energetic burden of viviparity without concomitant reductions in offspring body size. Although viviparous lizards are more prevalent in cold environments, transitions in thermal habitat only weakly predict parity mode evolution. Likewise, only cold tolerance adapts rapidly to thermal environment. Heat tolerance and preferred body temperatures track the thermal environment, but with a lag at million-year timescales. This lag likely reflects behavioral buffering: viviparous lizards thermoregulate to low body temperatures, regardless of ambient conditions. Rather than representing an adaptation to cold climates, the lower thermal and metabolic physiology of viviparous species are likely an energetic adjustment for reproduction that facilitated their prolific colonization of cooler environments.
\end{abstract}




\section{Introduction}

Live-bearing (viviparity) is a major evolutionary novelty in the tree of life. Viviparity is a parental care strategy that enhances physiological control and protection of developing embryos, providing higher offspring survivorship ${ }^{1,2,3,4}$. The evolution of viviparity within animals is prolific, with at least 160 independent origins, particularly in squamate reptiles (>100 origins $)^{5,6,7}$. Complete embryogenesis within the female reproductive tract is accompanied by physiological and reproductive adjustments: compared to oviparous counterparts, viviparous species often exhibit lower metabolic rates ${ }^{8}$ and reduced production (understood as energy allocated into reproduction) $)^{9}$. Although the transition from egg-laying to live-bearing has repeatedly arisen, the evolutionary pathways guiding metabolic and reproductive adjustments in viviparous lineages remain unclear.

Mass-specific metabolic rate is affected by both body mass and body temperature (see equation in Methods) ${ }^{10,11}$. These two traits provide three different combinations that could result in a lower ratio by which energy is acquired and allocated into survival, growth, and reproduction ${ }^{10}$ in viviparous species (Table 1a). In another way, mass-specific production is determined by the trade-off between offspring mass and annual fecundity (the number of hatchlings or eggs per year), normalized by maternal body mass ${ }^{9,12}$. Thus, three phenotypic pathways could explain the lower mass-specific production of viviparous species (Table 1b). Other combinations could result in a lower mass-specific metabolic rate or a lower mass-specific production, but will depend on the magnitude of each individual response. For example, if body mass and body temperature decrease in tandem, then the effect on mass-specific metabolic rate (either to increase or decrease) depends on the relative magnitudes of those shifts. 
Table 1. Three trait combinations could explain the lower mass-specific metabolic rate of viviparous species (a) and three other trait combinations could explain their lower massspecific production (b). Note that phrynosomatids are ancestrally oviparous and there are no back-transitions to oviparity. Therefore, our hypotheses are structured around explaining the transition to viviparity (rather than the other way around).

\section{a. Trait combinations resulting in a lower mass-specific metabolic rate}

i $\quad$ Body mass is similar among oviparous and viviparous species, but body temperature is lower in viviparous species.

ii Body mass is higher and body temperature is lower in viviparous species.

iii Body mass is higher in viviparous species, but body temperature is similar among oviparous and viviparous species.

\section{b. Trait combinations resulting in a lower mass-specific production}

iv Offspring size is similar between oviparous and viviparous species, but annual fecundity is lower in viviparous species.

$v \quad$ Offspring size and annual fecundity are lower in viviparous species.

vi Offspring size is smaller in viviparous species, but annual fecundity is similar between oviparous and viviparous species. 
The repeated evolution of viviparity among closely-related species provides a naturally replicated framework in which to test for signatures of adaptation. Squamate reptiles (lizards and snakes) account for $75 \%$ of the evolutionary origins of viviparous vertebrates $^{6}$. Here, we leveraged the prolific evolution of viviparity in phrynosomatid lizards, a lineage renowned for repeated transitions to live birth ${ }^{13}$, to investigate the evolutionary dynamics of behavior, physiology, morphology, and life history associated with viviparity. This diverse lizard family is comprised of 163 species distributed from North to Central America, and at elevations ranging from sea level to nearly 5,000 meters $^{13,14,15}$. Here, we addressed three goals. First, we tested whether in the transition from oviparity to viviparity, mass-specific metabolic rate and mass-specific production lower simultaneously, or whether these features can vary independently of one another in viviparous lineages. Second, we elucidated the evolutionary adjustments between body mass and body temperature resulting in the shift to lower mass-specific metabolic rate of viviparous species (Table 1a). Third, we clarified the combinations of offspring mass and offspring number (annual fecundity) resulting the shift to lower mass-specific production of viviparous species (Table 1b).

We began by building a phylogeny for phrynosomatid lizards (Supplementary Fig. 1) and reconstructing parity mode across the tree. Our reconstructions strongly support five independent shifts to live-bearing in phrynosomatids (Fig. 1a; Supplementary Fig. 2). We then assembled a dataset of adult body mass, adult body size (snout-vent length; SVL), thermoregulatory behavior (field-estimated body temperatures, laboratory preferred temperatures, and field-measured thermoregulatory effectiveness), thermal physiology (critical thermal limits), metabolic physiology (mass-specific metabolic rate), and life history traits (offspring mass, offspring size, clutch/litter size, and annual mass-specific 
production) by gathering newly collected and previously published data from 125 phrynosomatid species (80 oviparous and 45 viviparous species) (Supplementary Data 2). To connect phenotypic variation to the local thermal environment, we also included estimates of mean annual temperature, mean temperature of the warmest quarter, and mean temperature of the coldest quarter for each species. Our dataset encompasses $77 \%$ of phrynosomatids, and includes representatives from all viviparous sub-lineages. We then fitted a series of evolutionary models to the behavioral, physiological, morphological, and reproductive data to determine the phenotypic dynamics associated with shifts from oviparity to viviparity. To contextualize these results, we explore the relationships between thermoregulatory behavior and environmental variables using evolutionary regressions.

\section{Results and Discussion}

Our evolutionary models confirm that the transitions from egg-laying to live birth are consistently associated with tandem reductions in the phenotypic optimum $(\theta$ parameter; see Methods) for mass-specific metabolic rate and mass-specific production (Fig. 1b). Compared with egg-laying species, shifts to viviparity induced an 1.8-fold reduction in the optimal mass-specific metabolic rate and a 2.4-fold reduction in optimal annual production (Supplementary Table 2). In other words, viviparity in phrynosomatids represents a multidimensional phenotype in which the ratio and quantity of energy allocated into maintenance and reproduction is decreased.

Our results can be supported via a combination of metabolic and life history theory ${ }^{10,12,16,17}$. In low-predation environments, populations evolve toward a lower metabolic rate and lower reproductive allotment ${ }^{16}$. Species with lower mass-specific metabolic rate also exhibit reduced mass-specific production, and are positioned on the 
slow end of the fast-slow life history continuum ${ }^{17}$. Given these premises and assuming steady-state populations-populations in which energy invested into production (birth rates) equals energy lost by predation (death rates) $)^{10,12,18}$ — we infer that viviparity in phrynosomatids is a high-survivorship, low-fecundity phenotype positioned on the slow end of the fast-slow continuum. This notion is supported by the inherent maternal protection and mortality reduction afforded by in utero embryonic development (in comparison to eggs laid in nests) against abiotic and biotic hazards $2,3,4,19,20,21,22$ and with the common distribution of viviparous species in colder environments such as high elevation $^{13,22}$, where predation risk for ectotherms is lower ${ }^{23,24,25}$. Whereas viviparous females can replace themselves each generation by allocating less energy into maintenance and production (normalized by body mass), oviparous females must instead expend greater energy into producing more offspring per year (Fig. 1b). 


\section{a. Evolutionary Transitions to Live Birth}

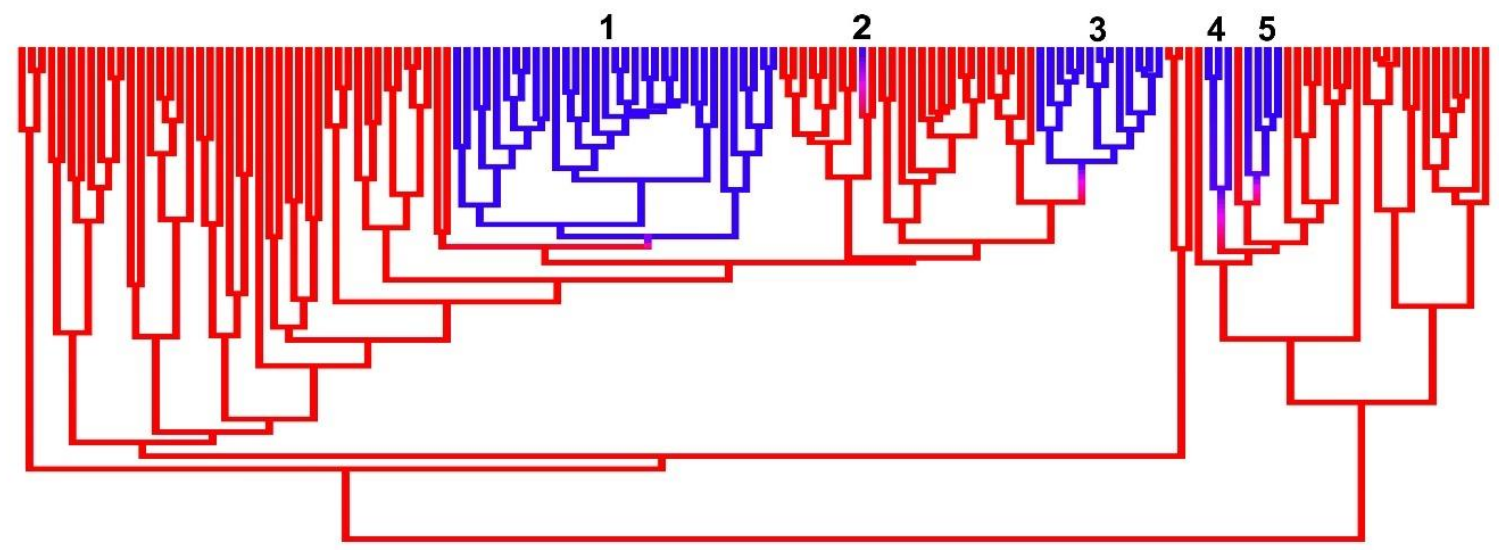

b. Evolutionary Optimal Trait Values $(\theta)$

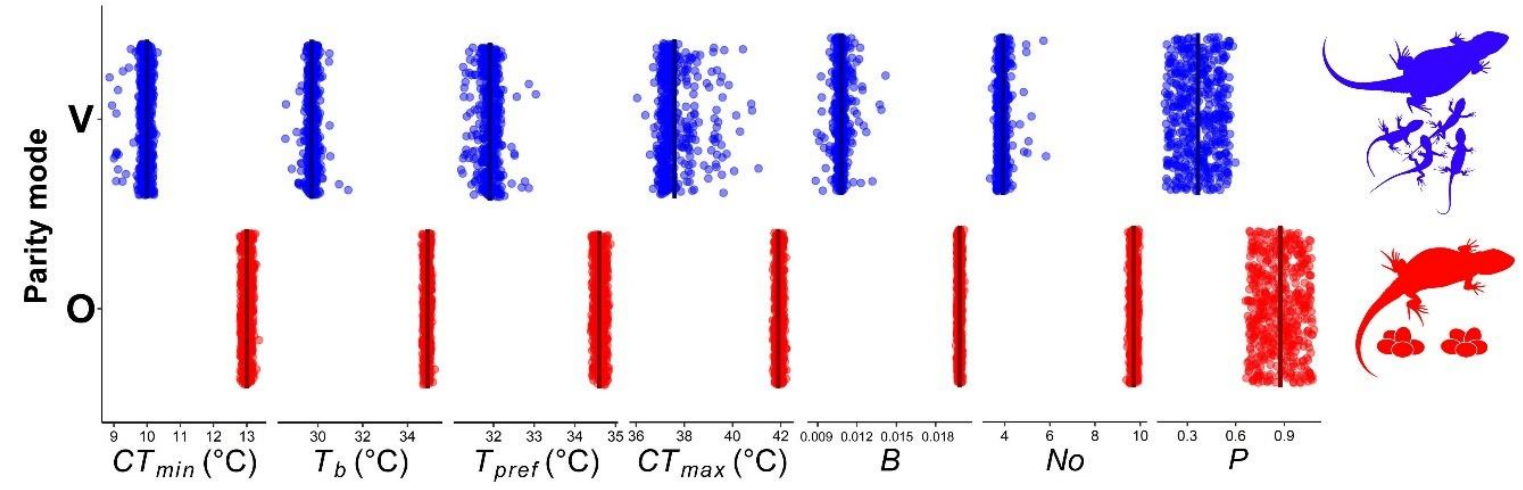

Fig. 1 Parallel reductions in physiological and life history traits are associated with

viviparity in phrynosomatid lizards. a Five evolutionary transitions from egg-laying (red) to live-bearing (blue) occurred in phrynosomatids. b Viviparous lineages are characterized by reductions in the evolutionary optimal trait values $(\theta)$ for cold tolerance $\left(C T_{\min }\right)$, fieldestimated body temperature $\left(T_{b}\right)$, the laboratory-measured preferred body temperature $\left(T_{\text {pref }}\right)$, heat tolerance $\left(C T_{\max }\right)$, mass-specific metabolic rate $(B)$, the annual number of offspring $(\mathrm{No})$, and mass-specific production $(P)$. Evolutionary optimal trait values $(\theta)$ were inferred from the Ornstein-Uhlenbeck (OU) model-fitting procedure (see Methods). Each point represents a different stochastic character map from our analyses ( $n=500$ per trait). 


\section{Pathways for a reduced mass-specific metabolic rate}

Reductions in mass-specific metabolic rate might reflect different evolutionary pathways involving changes in body mass, body temperature, or both (Table 1a). We do not find any support for adaptive shifts in body mass associated with viviparity in phrynosomatids (Supplementary Table 2). Phylogenetically-corrected body size (SVL), which is strongly correlated with body mass $\left(\log _{10}\right.$ body mass $=0.288 \log _{10} \mathrm{SVL}+1.522, p=0$; Supplementary

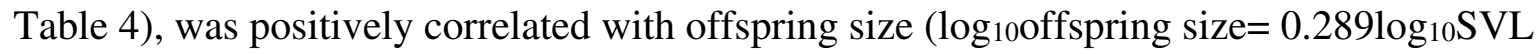
$+0.906, p<0.001$; Fig. 2a; Supplementary Table 4$)$ and litter/clutch size ( $\log _{10}$ litter or clutch size $=1.132 \log _{10} \mathrm{SVL}-1.236, p<0.001$; Fig. 2b; Supplementary Table 4). Therefore, whether viviparous or oviparous, larger females produce larger neonates, and are more fecund. Correspondingly, we infer that during the transition to live birth in phrynosomatids, the evolutionary optimal body size in females ${ }^{26}(\theta=61.1 \mathrm{~mm}$ SVL; Supplementary Table 2$)$ remains unchanged because any size shift also affects the quality and quantity of offspring.
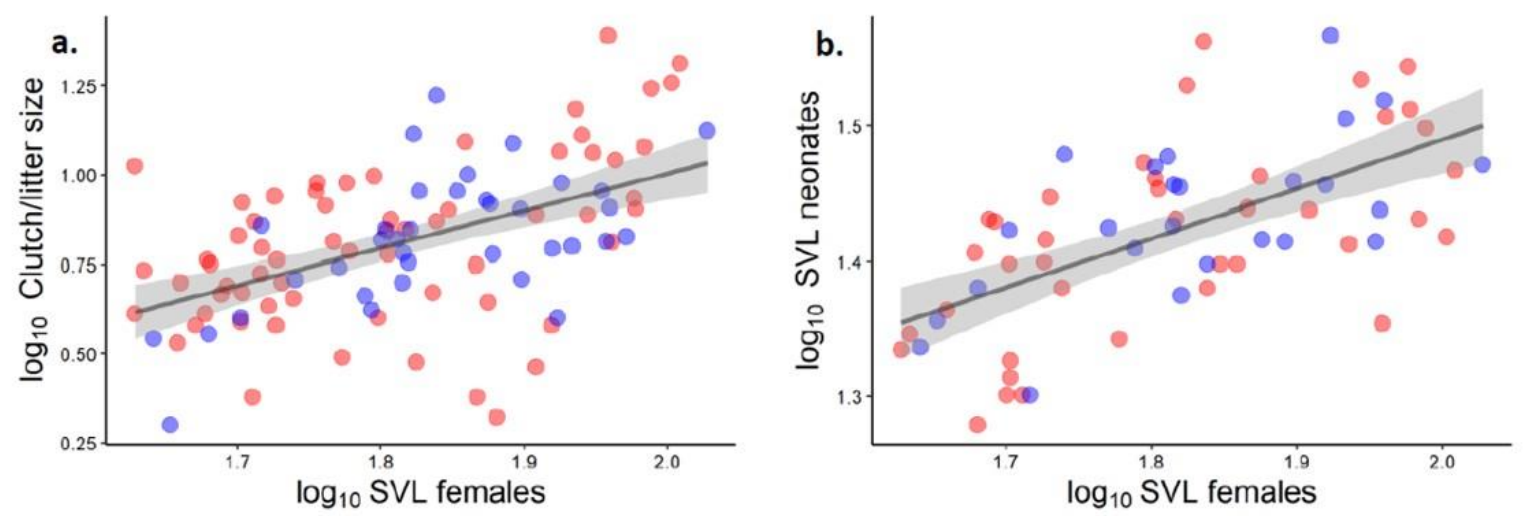

Fig. 2 Influence of dam's body size on clutch/litter size $(\mathbf{a} ; \mathrm{y}=1.1321 \mathrm{x}-1.2362, p<0.01)$ and on the body size of neonates $(\mathbf{b} ; \mathrm{y}=0.2893 \mathrm{x}+0.9064, p<0.01)$ in phrynosomatid lizards. Blue circles correspond to viviparous species whereas red circles correspond to oviparous species. 95\% confidence bands are included around regression lines. 
In contrast to body mass, however, transitions to viviparity are associated with a $5^{\circ} \mathrm{C}$ reduction in the optimal body temperature $\left(\theta=29.7^{\circ} \mathrm{C}\right)$ when compared to their egglaying counterparts $\left(\theta=34.9^{\circ} \mathrm{C}\right.$; Fig. 1b; Supplementary Table 2). Therefore, reductions in core temperature, but not body mass, characterize the transition to live birth in Phrynosomatidae (corresponding to hypothesis $\boldsymbol{i}$ in Table 1a). Three non-mutually exclusive hypotheses could explain the lower body temperatures of viviparous species ${ }^{27}$. First, during pregnancy (and especially during late pregnancy), body mass increases and females tend to reduce their body temperature ${ }^{28,29}$. This adjustment allows pregnant females to maintain a lower mass-specific metabolic rate. Second, incubation temperature influences the phenotype and survivorship of developing embryos ${ }^{3,30}$. The optimal temperature for embryonic development in nests ${ }^{31}$ tends to be lower than the optimal body temperature of oviparous adults $\left(\theta=34.9^{\circ} \mathrm{C}\right)$. Correspondingly, it is possible that in the transition to live birth, selection favors a lower preferred body temperature, which could better match the optimal temperature for intrauterine embryonic development. These two possibilities are not mutually exclusive and could operate in tandem. Therefore, the lower body temperatures (and lower mass-specific metabolic rate) could metabolically compensate for longer gestation length, optimize embryonic development, and reduce embryonic mortality ${ }^{28,30}$. A third idea centers around physiological adjustments to cold environments. The disproportionate representation of viviparous species in cooler habitats ${ }^{13}$ could have resulted in adaptive shifts to a more cool-adjusted thermal physiology (e.g., lower cold tolerance $\left(C T_{\min }\right)$, heat tolerance $\left(C T_{\max }\right)$, preferred body temperature $\left(T_{\text {pref }}\right)$, and body temperature $\left.\left(T_{b}\right)\right)$. If this were true, then we would expect a strong evolutionary match between the local thermal environment and species' thermal physiological traits. 
Consistent with the hypothesis of cold-environment adaptation, we discovered that viviparous phrynosomatids also evolved a lower optimal cold tolerance $\left(\theta=9.95^{\circ} \mathrm{C}\right)$ than the oviparous species $\left(\theta=13{ }^{\circ} \mathrm{C}\right.$; Fig. 1b; Supplementary Table 2$)$. We also found a strong positive relationship between mean annual temperature and cold tolerance in both oviparous and viviparous species (Fig. 3a; Supplementary Table 4). This relationship is matched by a rapid pace of cold tolerance adaptation $\left(t_{1 / 2}=0\right.$ million year for viviparous and oviparous species; Supplementary Table 5). Thus, the reduced cold tolerance of viviparous species can be explained simply by their overrepresentation in cooler environments ${ }^{13}$. Enhanced cold tolerance in cooler environments, regardless of parity mode, fits into a broader picture of ecophysiological evolution in ectotherms. Specifically, cold tolerance is generally labile ${ }^{32}$ and often correlates tightly with the minimum temperatures ectotherms experience in their environments ${ }^{33}$. This tight connection often reflects limited opportunities to behaviorally buffer against the cold, which results in rapid cold adaptation to prevailing conditions ${ }^{34}$.

We also detected parallel evolutionary reductions in the phenotypic optimum for heat tolerance $\left(\theta=37.6{ }^{\circ} \mathrm{C}\right.$ for viviparous and $41.9^{\circ} \mathrm{C}$ for oviparous species; Fig. $\mathbf{1 b}$; Supplementary Table 2). However, heat tolerance is uncorrelated with the thermal environment (Fig. 3d; Supplementary Table 4), in turn reflecting a much longer phylogenetic half-life for this trait $\left(t_{1 / 2}=8.8\right.$ million years for viviparous, and $t_{1 / 2}=17.8$ million years for oviparous species; Supplementary Table 5). In addition to thermal limits, the preferred body temperature is lower in viviparous species than in oviparous species $(\theta=$ $31.9^{\circ} \mathrm{C}$ for viviparous and $34.6^{\circ} \mathrm{C}$ for oviparous species; Fig. 1b; Supplementary Table 2). Yet, mean annual temperature is a strong predictor of preferred body temperature only in oviparous species (Fig. 3c; Supplementary Table 4). Likewise, the preferred body 
temperature in oviparous species evolves rapidly in response to shifts in mean annual temperature $\left(t_{1 / 2}=0.8\right.$ million years; Supplementary Table 5$)$, whereas the pace of $T_{\text {pref }}$ adaptation is considerably slower in viviparous species $\left(t_{1 / 2}=13.8\right.$ million years; Supplementary Table 5). Together, these results imply that reductions in $C T_{\max }$ and $T_{\text {pref }}$ in viviparous species are at least partially decoupled from shifts in the thermal environment itself. Rather, we suspect that viviparous species are cold-adapted for reasons besides their prevalence in colder habitats.

Consistent with this idea, the field-measured body temperature of viviparous species is poorly correlated with mean annual temperature (Fig. 3b), or with any of our environmental variables (Supplementary Table 4). In contrast, mean annual temperature is positively correlated with body temperature in oviparous lizards (Fig. 3b), although the strength of the relationship is weak. Correspondingly, body temperature exhibits a slow pace of adaptation with respect to mean annual temperature in phrynosomatids $\left(t_{1 / 2}=8.5\right.$ million years for viviparous species, and $t_{1 / 2}=13.8$ million years for oviparous species; Supplementary Table 5). Whether found in relatively cool habitats or in relatively warm habitats (Supplementary Fig. 3), viviparous lizards generally exhibit a lower core temperature than their oviparous counterparts. Yet, regardless of parity mode, thermoregulatory effectiveness (the ability to maintain core temperature within the preferred range $)$ is indistinguishable among parity modes in these lizards $\left(F_{1,62}=0.112\right.$, $p=0.9$; mean $E=0.8 \pm 0.02 S E$ for both parity modes), meaning that all phrynosomatids are relatively effective thermoregulators. Even when viviparous species are found in warm habitats and oviparous species are found in cool habitats, lizards behaviorally maintain their body temperature within (or close to) their respective preferred thermal ranges. 

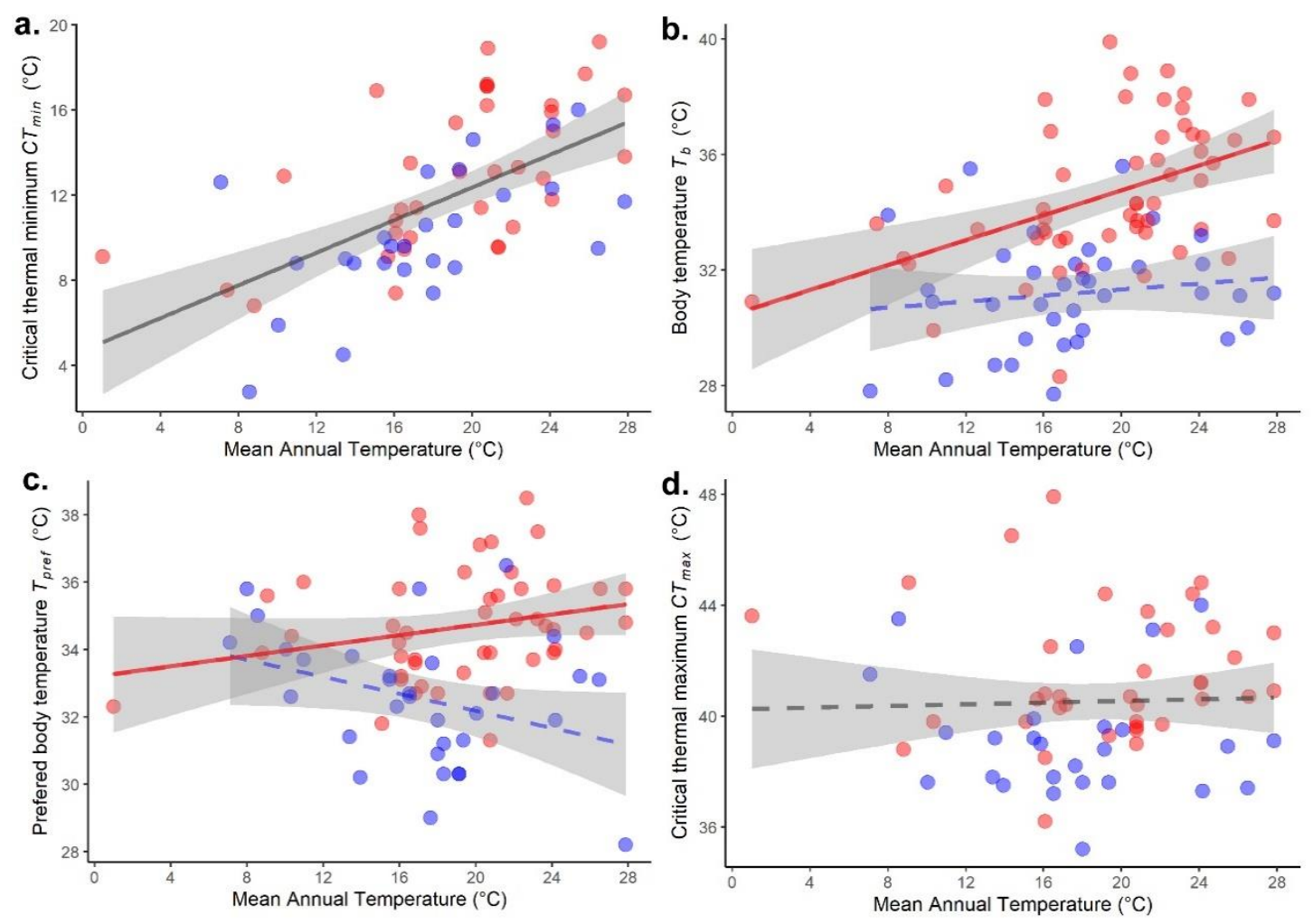

Fig. 3 Influence of mean annual temperature (MAT) on cold tolerance $(\mathbf{a} ; y=0.354 x+$ 5.639, $p=0$;), field-body temperature (b; for oviparous: $\mathrm{y}=0.230 \mathrm{x}+30.3, p=0$, and for viviparous: $\mathrm{y}=0.055 \mathrm{x}+30.69, p=0.4)$, preferred body temperature (c; for oviparous: $\mathrm{y}=0.094 \mathrm{x}+32.78, p=0.03$, and for viviparous: $\mathrm{y}=-0.078 \mathrm{x}+34.6, p=0.2)$, and heat tolerance $(\mathbf{d} ; \mathrm{y}=0.032 \mathrm{x}+41.29, p=0.5)$. Blue circles correspond to viviparous species whereas red circles correspond to oviparous species. Solid lines represent slopes with statistical significance $(<0.05)$, and dashed lines represent slopes that are not statistically different from $0.95 \%$ confidence bands are included around regression lines. PGLS results using the other macroclimatic predictors (bio10 and bio11) are given in Supplementary Table 4. Parity mode did not impact the relationship between MAT and $C T_{\min }$ (a) or $C T_{\max }(\mathbf{d})$; therefore, data were combined for oviparous and viviparous species (as indicated by a single gray regression line). 
Furthermore, under the threshold model, we found low evolutionary covariation between environmental predictors and reproductive mode (mean annual temperature: $r=$ 0.205 ; mean temperature of the coldest quarter: $r=-0.001$; mean temperature of the warmest quarter: $r=-0.359)$, meaning that shifts in the thermal environment are not strongly associated with evolutionary transitions in parity mode. Given these results, we infer that viviparity is not an adaptation to cold climate per se; rather, a cool-adjusted thermal and metabolic physiology might have afforded viviparous species enhanced access into cooler environments. The notion that physiological evolution in viviparous lizards reflects life history adaptation co-opted to life in cold environments echoes ideas dating back more than half a century ago ${ }^{35}$, but which lacked the behavioral, physiology, and life history trait data necessary for rigorous phylogenetic examination.

\section{Lower fecundity reduces mass-specific production in viviparous lizards}

Reductions in mass-specific production might reflect different combinations of offspring mass and annual fecundity (Table 1b). Yet, the optimal offspring mass $(\theta=0.83 \mathrm{~g}$; Supplementary Table 2$)$ and offspring size $(\theta=26.4 \mathrm{~mm}$ snout-vent-length; Supplementary Table 2) do not vary between viviparous and oviparous species. The retention of optimal offspring size $\mathrm{e}^{36}$ and mass in viviparous lineages could reflect the presence of a shared adaptive optimum; smaller or larger offspring sizes (based on maternal morphology), typically exhibit reduced survivorship ${ }^{37,38}$. Compared with viviparous species, oviparous phrynosomatids produce $~ 2.5$-fold more offspring per year (Fig. 1b; Supplementary Table

2). The lower annual fecundity and consequent lower mass-specific production in viviparous species could reflect their relatively long gestation periods, which limit most species to a single litter per year ${ }^{9,39}$. Therefore, the available evidence indicates that in the 
evolutionary transition to viviparity, selection favors allocating less energy into production. Because the pathway to this phenotype involves only downshifts in annual fecundity, offspring size is not compromised (corresponding to hypothesis $\boldsymbol{i} \boldsymbol{v}$ in Table $\mathbf{1 b}$ ).

The parallel evolution of viviparity in phrynosomatid lizards is accompanied by parallel phenotypic shifts in metabolic physiology, thermal physiology, thermal behavior, and fecundity. Tandem reductions in thermal physiology and fecundity clearly reduce the energetic burden of pregnancy without simultaneously impact offspring quality. Yet, even with these reductions, viviparous phrynosomatids are restricted to a single litter per year ${ }^{39}$. Given that neonate size is comparable among parity modes, viviparous species are likely straddling an evolutionary tight-rope between the competing fitness demands of growth/maintenance and fecundity. The disproportionate presence of viviparous species in cold environments has fueled a decades-long interpretation of viviparity as a physiological response to lower ambient temperatures ${ }^{22}$, as opposed to a phenotype that can readily be coopted to life in cold environments. While the inference space of our results is limited to phrynosomatid lizards, the general principles that explain evolutionary patterns in this system also characterize other ectotherm lineages, which account for nearly all origins (and species diversity) of viviparity in animals. We suspect, therefore, that the patterns we observed here might be generalizable across the animal tree of life.

\section{Methods}

Phylogeny and divergence time estimation. To estimate the phylogeny and divergence time among phrynosomatid species we used sequences of five mitochondrial and eight nuclear genes available in GenBank for 149 taxa (Supplementary Data 1). Accession

numbers were the same as those used in Martínez-Méndez et al. ${ }^{40}$ for the Sceloporus 
torquatus, S. poinsettii and S. megalepidurus groups and the same as those in Wiens et al. ${ }^{41}$ for other phrynosomatid species. For taxa not included in the previous references, we searched GenBank for available sequences. We then performed alignments for each gene using MAFFT ver. $7^{42}$ and concatenation and manual refinement using Mesquite ver. $3.6^{43}$; obtaining a concatenated matrix of 9,837 bp for 149 taxa (the block dataset is available in the file entitled "Phrynosomatidae_Gene_Matrix.nex", which is available in the Supplementary Material). For the relaxed clock analyses, three nodes were calibrated using lognormal distributions based on two previous studies ${ }^{41,44}$. The first calibration was set for the Sceloporus clade (offset 15.97 million years ago (MYA)) based on a fossil Sceloporus specimen $^{45}$ ). The second calibration point was set for the Phrynosoma clade (offset 33.3 MYA) based on the fossil Paraphrynosoma greeni ${ }^{46}$, and the last calibration point was for the Holbrookia-Cophosaurus stem group (offset 15.97 MYA) given the fossil Holbrookia antiqua ${ }^{45}$. We conducted dating analysis with the concatenated sequences matrix, partitioned the mitochondrial and nuclear information, each gene under GTR $+\mathrm{I}+\Gamma$ model, and allowed independent parameter estimation. We performed Bayesian age estimation with the UCLN model in BEAST version 2.5.2 $2^{47}$ and run on CIPRES ${ }^{48}$. Tree prior (evolutionary model) was under the Birth-Death model, and we ran two MCMC analyses for 100 million generations each and stored every 20,000 generations. We assessed convergence and stationarity of chains from the posterior distribution using Tracer version $1.7^{49}$. We combined independent runs using LogCombiner (version 2.5.2; BEAST distribution) and discarded $30 \%$ of samples as burn-in, obtaining values of effective sample size (ESS) greater than 200. We estimated the maximum clade credibility tree from all post-burnin trees using TREEANOTATOR ver.1.8.4 ${ }^{49}$. The ultrametric tree is available as a supplementary file "Phrynosomatidae_Tree.tre" (in the Supplementary Material). As we 
describe below, we accounted for phylogenetic uncertainty in our models by re-performing analyses using 500 trees that we randomly sampled from our posterior distribution. The 500 sampled trees are available as a supplementary file "Phrynosomatidae_500_Trees.tre" (in the Supplementary Material).

Data collection. Parity mode. We categorized each species as either oviparous or viviparous based on previously published databases ${ }^{13,39,50,51}$, published references, and unpublished data (Supplementary Data 2). Our assignations align with other studies, except for one species, Sceloporus goldmani, which has been previously considered a viviparous species $^{13,52}$. The only available sequence in GenBank (U88290) for that species is from a male (MZFC-05458) collected in Coahuila, Mexico ${ }^{52}$. However, in that same locality, one of us (F. R. Méndez-de la Cruz; unpubl. data) collected two females of the same species and both laid eggs. Thus, the population of S. goldmani herein included is considered oviparous.

Thermal physiology. We compiled a database of four thermal physiological traits that influence the performance and fitness of ectotherms ${ }^{53}$ for 104 phrynosomatid species. These data were gathered from both published sources and from our own field and laboratory work (Supplementary Data 2). The thermal physiological traits we examined were the field body temperature $\left(T_{b}\right)$ of active lizards ${ }^{33}$, the preferred body temperature ( $\left.T_{\text {pref }}\right)$ in a laboratory thermal gradient ${ }^{54}$, cold tolerance (critical thermal minimum, $C T_{\min }$ ) and heat tolerance (critical thermal maximum, $\left.C T_{\max }\right)$. These latter two traits $\left(C T_{\min }\right.$ and $\left.C T_{\max }\right)$ describe the thermal limits of locomotion; specifically, they describe the lower and upper temperatures, respectively, at which lizards fails right itself when flipped onto their 
backs $^{55,32}$. To minimize the confounding effects of experimental design, we limited our data selection to those that were measured with similar methods. Correspondingly, our data collection approach mirrored that of the published studies from which we extracted data. If species were measured from multiple populations, we summarized physiological traits using means weighted by sample size, and also similarly weighted environmental variables. In cases where locality details, but not coordinates, were available, we georeferenced sampling sites using Google Earth (Version 7.3.3). All physiological data correspond only to adult lizards. Some studies have found that pregnant females reduce their core temperature to better match the optimal incubation temperature for their offspring ${ }^{28}$. When we detected effects of reproductive condition on thermal physiology, we excluded data from pregnant (or gravid) females. To test whether physiological traits differed between sexes, we performed $t$-tests for a sub-set of 25 species (Supplementary Table 1). We did not find significant thermal physiological differences between (non-gravid/non-pregnant) females and males in $T_{b}(t=0.172, d f=48, p=0.86), T_{\text {pref }}(t=-0.482, d f=48, p=0.63), C T_{\text {min }}$ $(t=0.742, d f=45, p=0.46)$ or $C T_{\max }(t=-0.407, d f=42, p=0.69)$, so we combined data for both sexes. Ideally, we would re-run all analyses using thermal trait data from gravis/pregnant females, but such data are still lacking. Given that, in the few cases where robust data do exist, preferred temperatures in pregnant females tend to be even lower than in nonpregnant females ${ }^{29}$, we suspect that our analyses provide a relatively conservative estimate of physiological differences among parity modes.

Operative temperatures. As we describe below, we were interested in estimating thermoregulatory patterns among phrynosomatid species. Doing so requires knowledge of the environmental operative temperatures $\left(T_{e}\right)$ available to lizards. $T_{e}$ represents the 
equilibrium temperature of an animal in the absence of behavioral thermoregulation ${ }^{56}$. We recorded $T_{e}$ using previously-calibrated pipe models (made of polyvinylchloride), which were similar in shape, size, and heat gain/loss with respect to lizards of each species ${ }^{33}$. Into each pipe model, we inserted one temperature data logger (Thermochron iButton; model DS1921G), which recorded temperature $\left( \pm 0.1^{\circ} \mathrm{C}\right)$ every ten minutes during the same periods during which we were measuring field-active body temperatures $\left(T_{b}\right)$ in lizards. Operative temperatures were measured during a sampling period of 1-5 days for each locality, which always occurred during times of year when lizards exhibit surface activity.

Thermoregulatory effectiveness. Several studies have found that viviparous species exhibit lower body temperatures than their oviparous counterparts ${ }^{27}$. Less well known, however, is whether lower body temperatures reflect a behaviorally passive property of viviparous lizards, perhaps because of their distributions in relatively cooler habitats, or whether those low body temperatures reflect a more behaviorally active decision to thermoregulate to a cooler temperature. Therefore, we were particularly interested in the thermoregulatory patterns of oviparous and viviparous species. We calculated the effectiveness of temperature regulation $(E)$, a ratio that describes how well lizards maintain their $T_{b}$ within their $T_{\text {set }}$ range (central $50 \%$ of data of $T_{p r e f}, T_{\text {set } 25}$ and $\left.T_{\text {set }} 75\right)$, given the operative temperatures $\left(T_{e}\right)$ available in their habitat ${ }^{54}$. We estimated $E$ for each species following the equation proposed by Hertz et al. ${ }^{54}$ :

$$
E=1-(\overline{d b} / \overline{d e})
$$

where $\overline{d b}$ is the average of the accuracy of body temperature, and indicates the deviation of $T_{b}$ from $T_{\text {set }}$ range. If each $T_{b}<T_{\text {set } 25}$, then each $d b=T_{\text {set } 25-} T_{b}$, if each $T_{b}>T_{\text {set } 75}$, then each $d b=$ 
$T_{b}-T_{\text {set75}}$, and if each $T_{b}$ is within $T_{\text {set }}$ range, then each $d b=0 . \overline{d e}$ is the average of thermal quality of the habitat, and indicates the deviation of $T_{e}$ from $T_{\text {set }}$ range. If each $T_{e}<T_{\text {set25, }}$, then each $d e=T_{\text {set25 }}-T_{e}$, if each $T_{e}>T_{\text {set75, }}$, then each $d e=T_{e}-T_{\text {set75, }}$, and if each $T_{e}$ is within $T_{\text {set }}$ range, then each $d e=0$. Values of $\overline{d b}$ close to 0 indicate that individuals accurately maintain their body temperature within their preferred range, and values of $\overline{d e}$ close to 0 indicate that the habitat temperatures approximate (and/or fall within) the preferred range of individuals. As both $\overline{d b}$ and $\overline{d e}$ increase, body temperatures and operative temperatures, respectively, exceed species' preferred thermal ranges. As such, $E$ values close to 1 indicate that lizards are highly effective thermoregulators, and $E$ values close to 0 indicate that individuals are more behaviorally passive with respect to thermal environment. $E$ was only estimated in cases where $T_{e}$ and $T_{b}$ were sampled during the same period, and if $T_{s e t}$ was measured from the same population of lizards from which $T_{b}$ was measured. In total, we were able to gather estimates of $E$ from 64 species (37 oviparous and 27 viviparous) of phrynosomatid lizards.

Environmental temperature. In addition to the operative temperatures, which provided a detailed (but temporally limited) snapshot of thermal environment, we gathered data on general thermal trends for each species' habitat. Specifically, we also gathered climatic measurements for each locality (Supplementary Data 2) from which any lizard trait data were gathered by extracting thermal variables from the environmental layers available in the WorldClim dataset (resolved to approximately $\left.1 \mathrm{~km}^{2}\right)^{57}$. These variables were mean annual temperature (bio1), mean temperature of the warmest quarter (bio10), and mean temperature of the coldest quarter (bio11). We did not use these data to calculate $T_{e}$ for estimates of thermoregulatory effectiveness (as $E$ should be calculated from $T_{e}$ measured 
during the same time period as $T_{b}$ ). Instead, we used these bioclimatic variables as predictors of phenotypic trait variation using evolutionary regressions as described below.

Morphology and life-history traits. We gathered published and unpublished information for mean snout-vent length (SVL; mm), a common measure of body size in squamates, and body mass (g) of adult females and neonates. We also recorded clutch or litter size (i.e., the number of offspring produced per reproductive bout), and the number of clutches or litters produced during one year (Supplementary Data 2). We multiplied these two last traits to quantify annual fecundity, which reflects the total predicted annual reproductive output of a given species. We used annual fecundity for three reasons. First, in phrynosomatids (with exception of some populations of three species ${ }^{58,59,60}$ ), females have annual (seasonal) patterns of reproduction ${ }^{39}$. Second, oviparous species are able to produce eggs in multiple clutches per year ${ }^{61}$, whereas viviparous species are typically able to produce only one litter in the same unit time ${ }^{39}$. Indeed, viviparous species tend to produce only one litter per year regardless of reproductive window length. For example, both Phrynosoma hernandesi, a species with shorter gestation (3 months) ${ }^{62}$ and Sceloporus bicanthalis, a species with continuous reproduction $^{59,63}$, produce a single litter per year. Third, the maximum lifespan for phrynosomatid lizards varies considerably, but does not differ between parity modes ${ }^{36}$. For some species, the lifespan is typically $\sim 1$ year (documented for the oviparous species, Sceloporus aeneus, and the viviparous species, Sceloporus bicanthalis ${ }^{59,64}$ ), whereas the maximal lifespan is $\sim 10$ years (documented for the oviparous species Phrynosoma asio ${ }^{65}$ and for the viviparous species Sceloporus macdougalli ${ }^{66}$ ). Thus, consistent with other studies $^{17,51}$, we consider that by standardizing production to one year, we have an estimation of reproductive output that can be readily compared among parity modes. 
Mass-specific metabolic rate. We estimated individual metabolic rate $(I)$ for female lizards following the equation proposed by Brown et al. ${ }^{10}$ :

$$
I=i_{0} M^{3 / 4} e^{-E / k T}
$$

where $i_{0}=$ is a normalization constant, $M$ is mean body mass $(\mathrm{g})$ of females, $e=$ Euler's number, $E=$ activation energy, $k=$ Boltzmann's constant, and $T=$ field body temperature (in Kelvin). Then, $I / M$ can be used to obtain mass-specific metabolic rate $(B)$. SVL of phrynosomatids lizards is more frequently reported that body mass. We built a data base of body mass and SVL of adult females for 30 phrynosomatid species (none were gravid or pregnant) via a combination of unpublished and published information (Supplementary Data 2). Using these data, we built a non-phylogenetic equation to predict $\log _{10}$ body mass from $\log _{10}$ SVL. Our equation is $\log _{10}$ body mass $=3.355 \log _{10} \mathrm{SVL}-5.065\left(R^{2}=0.88\right.$, $\mathrm{P}<0.001$ ). Then, we transformed the $\log _{10}$ body mass value to an integer value (body mass= $\left.10^{\log 10 \mathrm{body} \text { mass }}\right)$. With our equation, we predicted the body mass of females for species for which SVL and field body temperature were available. Based on this approach, we obtained a total database of mass-specific metabolic rate of females for 96 phrynosomatid species (Supplementary Data 2).

Mass-specific production. We estimated mass-specific production $(P)$ as the product of neonate mass and annual fecundity/female body mass ${ }^{9}$. Therefore, $P$ describes the amount of energy converted into reproductive effort per year, normalized by maternal body mass.

Evolutionary analyses. All evolutionary analyses were conducted using the R environment for statistical computing, version 3.6.0. ${ }^{67}$ 
Stochastic character mapping of parity mode. To estimate the number of transitions between parity modes we performed stochastic character mapping ${ }^{68}$ onto the ultrametric tree of Phrynosomatidae using the make.simmap function with 500 simulations and a transition model of equal rates (ER) in phytools R package ${ }^{69}$. We selected the ER model of character evolution because was it the best-supported model $(\triangle \mathrm{AICC}=0$, weight $=0.65)$ in comparison to a symmetrical model $(\mathrm{SYM} ; \mathrm{AICC}=2.1$, weight= 0.22$)$ and with an allrates-different model (ARD; $\triangle \mathrm{AICC}=3.2$, weight $=0.13)$.

Ancestral state reconstruction. To fit mean annual temperature through Phrynosomatidae tree and graphically show the thermal environment where each population of each species used in this study inhabits, we performed ancestral state reconstruction using contMap function in phytools R package ${ }^{69}$.

Phylogenetic analyses of variance (ANOVA). To test for differences in effectiveness of temperature regulation, we performed phylogenetic ANOVAs using the aov.phylo function with 500 simulations in phytools R package ${ }^{69}$.

Comparing trait evolution between viviparous and oviparous species. We were interested in whether transitions to viviparity are associated with predictable phenotypic shifts. To this end, we tested if parity mode ("oviparous" or "viviparous") was associated with different evolutionary patterns of mass-specific metabolic rate, mass-specific production, body mass and size, thermal physiological traits, and life history traits by fitting Brownian motion (BM) and Ornstein-Uhlenbeck (OU) models. To do so, we used the R package OUwie ${ }^{70}$ and the 500 stochastic character maps of parity mode built with the make.simmap function in the R package phytools ${ }^{69}$. We fitted three different models. The simplest (BM1) is a 
single-rate BM model in which a single rate of stochastic trait evolution $\left(\sigma^{2}\right)$ was estimated for all Phrynosomatidae. The other two models were all adaptive OU models that varied in whether the estimated phenotypic optimum $(\theta)$ was either constrained to be equal among parity modes (OU1), or allowed to vary between oviparous and viviparous species (OUM). We fitted these three models separately for each physiological trait $\left(T_{b}, T_{p r e f}, C T_{\min }, C T_{\max }\right.$, and mass-specific metabolic rate), each morphological variable (adult body mass, and adult body size), and each life history trait (offspring mass, offspring size, annual fecundity, and mass-specific production) (Supplementary Table 2). For these (and all) analyses, body mass, body size, offspring mass, offspring size and annual fecundity were $\log _{10}$ transformed. We assessed model fit using a modified Akaike information criterion $\left(\mathrm{AIC}_{\mathrm{C}}\right)$ that incorporates a correction for small size ${ }^{71}$. Our approach, which was based on 500 stochastic character maps derived from the MCC tree, allowed us to account for uncertainty in reconstruction across the preferred tree, but could not account for uncertainty in the phylogeny itself. Therefore, we also repeated our stochastic character mapping across 500 individually-sampled trees from the posterior distribution to account for this additional source of phylogenetic uncertainty, and repeated all of our OUwie analyses using these 500 sampled trees. Our results in this latter approach are comparable to those using the MCC tree (Supplementary Table 3). Therefore, we present our results from the analyses based on the MCC tree in the main document.

Phylogenetic Generalized Least Squares (PGLS). To know the evolutionary relationship between adult body mass and adult body size, clutch or litter size and adult body size, and offspring size and adult body size, we performed PGLS regressions using the $g l s$ function in the $\mathrm{R}$ package nlme $\mathrm{e}^{72}$. 
Threshold model for ancestral state reconstruction. We were interested in whether changes in the thermal environment were strong predictors of parity mode shifts. We tested for the evolutionary covariation between the thermal environment and reproductive mode (oviparous vs. viviparous) using the threshold model ${ }^{73,74}$ using threshBayes function in the phytools R package ${ }^{69}$. The threshold model is used to test for evolutionary covariation between continuous and discrete traits ${ }^{74}$. Under the threshold model, a discrete character (i.e. oviparity or viviparity) evolves as a function of a continuously varying feature (termed "liability"). When the value of "liability" crosses a certain threshold, the state of the discrete character evolves (i.e., a transition from oviparous to viviparous occurs) ${ }^{73,74}$. We ran threshBayes for $1.0 \times 10^{6}$ generations, sampling every 100 generations, and discarding the first $200 \mathrm{~K}$ generations as burn-in. We ran separate analyses for mean annual temperature (bio 1), mean temperature of the coldest quarter (bio 10), and mean temperature of the warmest quarter (bio 11).

Stochastic linear Ornstein-Uhlenbeck models. Our OUwie analyses revealed reductions in the phenotypic optimum ( $\theta$ parameter) for thermal traits in viviparous lizards (see Results and Discussion). Yet, it is unclear whether reductions in thermal physiology reflect adaptation to cool environments (given the greater representation of viviparous lineages at high elevation ${ }^{13}$ ) or, instead, reflect metabolic compensation for live birth (hypothesis $i$ in Table 1a), which could be readily co-opted for life in cold environments. If a cool-adapted physiology reflects adaptation to cool environments, there should be a strong evolutionary association between the local thermal environment and thermal physiology. However, if a cool-adapted physiology reflects metabolic compensation for the heightened costs of pregnancy, then we expect viviparous lineages to exhibit a cool-adapted physiology 
regardless of ambient conditions, which should weaken the evolutionary relationship between the local thermal environment and corresponding thermal physiology.

To test these ideas, we used the SLOUCH model of Hansen et al. ${ }^{75}$, which simultaneously estimates an "evolutionary regression" and an "optimal" regression in an OU framework. The evolutionary regression describes the observed relationship between climatic predictors (mean annual temperature (bio 1), mean temperature of the warmest quarter (bio 10), and mean temperature of the coldest quarter (bio 11)) and physiological response variables $\left(C T_{\min }, T_{b}, T_{p r e f}\right.$, and $\left.C T_{\max }\right)$, while accounting for relatedness among species. The estimated "optimal regression", in contrast, describes the relationship between these variables predicted under an OU model, and assuming adaptation of the response variables to the predictor variables. In addition to the regressions, the model permits estimation of phylogenetic half-life $\left(t_{1 / 2}\right)$. Phylogenetic half-life represents the amount of time required for viviparous or oviparous lineages to get halfway to their thermal physiological optimum. So, a short $t_{1 / 2}$ (relative to the length of tree) indicates the phylogenetic signal degrades at a rapid pace. In contrast, a $t_{1 / 2}$ approaching (or exceeding) the length of the tree, indicates strong phylogenetic signal.

Similarity between the evolutionary and optimal regressions is supported when $t_{1 / 2}$ is close to 0 , which would indicate that transitions in the thermal environment are rapidly coupled with changes in thermal physiology. Differences in the slopes of these relationships, in contrast, are supported when the phylogenetic half-life $\left(t_{1 / 2}\right)$ of the model is bounded away from zero, implying phylogenetic inertia, or a lag in physiological adaptation to the thermal environment. Under this scenario, shifts in thermal environment are not strongly associated with shifts in thermal physiology. To run the analyses, we simultaneously estimated the evolutionary regression, optimal regression, and $t_{1 / 2}$ for each 
thermal physiological trait $\left(C T_{\min }, T_{b}, T_{\text {pref }}\right.$, and $\left.C T_{\max }\right)$ of phrynosomatid lizards, with respect to their thermal environment (bio1, bio10, and bio11) using an OU modeling framework in the $\mathrm{R}$ package SLOUCH${ }^{75}$.

\section{References}

1. Crespi, B. J. Facultative viviparity in a thrips. Nature 337, 357-358 (1989).

2. Wourms, J. P. \& Lombardi, J. Reflections on the evolution of piscine viviparity. Am. Zool. 32, 276-293 (1992).

3. Shine, R. A new hypothesis for the evolution of viviparity in reptiles. Am. Nat. 145, 809-823 (1995).

4. Furness, A. I. \& Capellini, I. The evolution of parental care diversity in amphibians. Nat. Commun. 10, 1-12 (2019).

5. Blackburn, D. G. Viviparity and oviparity: Evolution and reproductive strategies. In Encyclopedia of reproduction (eds. Knobil, T. E. \& Neill, J. D.) (London, UK: Academic Press, 1999).

6. Blackburn, D. G. Evolution of vertebrate viviparity and specializations for fetal nutrition: A quantitative and qualitative analysis. J. Morphol. 276, 961-990 (2015).

7. Helmstetter, A. J. et al. Viviparity stimulates diversification in an order of fish. Nat. Commun. 7, 1-7 (2016).

8. Zhang, L., Guo, K., Zhang, G. Z., Lin, L. H. \& Ji, X. Evolutionary transitions in body plan and reproductive mode alter maintenance metabolism in squamates. $B M C$ Evol. Biol. 18, 45 (2018).

9. Meiri, S., Brown, J. H. \& Sibly, R. M. The ecology of lizard reproductive output. Glob. Ecol. Biogeogr. 21, 592-602 (2012). 
10. Brown, J. H., Gillooly, J. F., Allen, A. P., Savage, V. M. \& West, G. B. Toward a metabolic theory of ecology. Ecology 85, 1771-1789 (2004).

11. Gillooly, J. F., Brown, J. H., West, G. B., Savage, V. M. \& Charnov, E. L. Effects of size and temperature on metabolic rate. Science 293, 2248-2251 (2001).

12. Burger, J. R., Hou, C. \& Brown, J. H. Toward a metabolic theory of life history. Proc. Natl. Acad. Sci. USA. 116 26653-26661 (2019).

13. Lambert, S. M. \& Wiens, J. J. Evolution of viviparity: A phylogenetic test of the cold-climate hypothesis in phrynosomatid lizards. Evolution 67, 2614-2630 (2013).

14. Lemos-Espinal, J. A. \& Ballinger, R. E. Comparative thermal ecology of the highaltitude lizard Sceloporus grammicus on the eastern slope of the Iztaccihuatl Volcano, Puebla, Mexico. Can. J. Zool. 73, 2184-2191 (1995).

15. Uetz, P. \& Hošek, J. The reptile database. http://reptile-database.org/ (2020).

16. Auer, S. K., Dick, C. A., Metcalfe, N. B. \& Reznick, D. N. Metabolic rate evolves rapidly and in parallel with the pace of life history. Nat. Commun. 9, 1-6 (2018).

17. Healy, K., Ezard, T. H. G., Jones, O. R., Salguero-Gómez, R. \& Buckley, Y. M. Animal life history is shaped by the pace of life and the distribution of age-specific mortality and reproduction. Nat. Ecol. Evol. 3, 1217-1224 (2019).

18. Brown, J. H., Hall, C. A. S. \& Sibly, R. M. Equal fitness paradigm explained by a trade-off between generation time and energy production rate. Nat. Ecol. Evol. 2, 262-268 (2018).

19. Neill, W. T. Viviparity in snakes: Some ecological and zoogeographical considerations. Am. Nat. 98, 35-55 (1964).

20. Tinkle, D. W. \& Gibbons, J. W. The distribution and evolution of viviparity in reptiles. Misc. Publ. Museum Zool. Univ. Michigan 1-55 (1977). 
21. Andrews, R. M. Evolution of viviparity in squamate reptiles (Sceloporus spp.): A variant of the cold-climate model. J. Zool. 250, 243-253 (2000).

22. Shine, R. Evolution of an evolutionary hypothesis: A history of changing ideas about the adaptive significance of viviparity in reptiles. J. Herpetol. 48, 147-161 (2014).

23. Fox, S. F., Perea-Fox, S. \& Castro-Franco, R. Development of the tail autotomy adaptation in lizards under disparate levels of predation at high and low elevations in Mexico. Southwest. Nat. 39, 311-322 (1994).

24. Roslin, T. et al. Latitudinal gradients: Higher predation risk for insect prey at low latitudes and elevations. Science 356, 742-744 (2017).

25. Domínguez-Godoy, M. A., Hudson, R., Pérez-Mendoza, H. A., Ancona, S. \& Díaz de la Vega-Pérez, A. H. Living on the edge: Lower thermal quality but greater survival probability at a high altitude mountain for the mesquite lizard (Sceloporus grammicus). J. Therm. Biol. 94, 102757 (2020).

26. Oufiero, C. E. \& Gartner, G. E. A. The effect of parity on morphological evolution among phrynosomatid lizards. J. Evol. Biol. 27, 2559-2567 (2014).

27. Sinervo, B. et al. Erosion of lizard diversity by climate change and altered thermal niches. Science 328, 894-899 (2010).

28. Mathies, T. \& Andrews, R. M. Influence of pregnancy on the thermal biology of the lizard, Sceloporus jarrovi: Why do pregnant females exhibit low body temperatures? Funct. Ecol. 11, 498-507 (1997).

29. Díaz de la Vega-Pérez, A. H., Barrios-Montiel, R., Jiménez-Arcos, V., Bautista, A. \& Bastiaans, E. High-mountain altitudinal gradient influences thermal ecology of the Mesquite Lizard Sceloporus grammicus. Can. J. Zool. cjz-2018-0263 (2019).

30. Beuchat, C. A. Temperature effects during gestation in a viviparous lizard. J. Therm. 
Biol. 13, 135-142 (1988).

31. Noble, D. W. A., Stenhouse, V. \& Schwanz, L. E. Developmental temperatures and phenotypic plasticity in reptiles: A systematic review and meta-analysis. Biol. Rev. 93, 72-97 (2018).

32. Muñoz, M. M. et al. Evolutionary stasis and lability in thermal physiology in a group of tropical lizards. Proc. R. Soc. B 281, 20132433 (2014).

33. Domínguez-Guerrero, S. F. et al. Interactions between thermoregulatory behavior and physiological acclimatization in a wild lizard population. J. Therm. Biol. 79, 135-143 (2019).

34. Muñoz, M. M. \& Bodensteiner, B. L. Janzen's hypothesis meets the Bogert effect: Connecting climate variation, thermoregulatory behavior, and rates of physiological evolution. Integr. Org. Biol. 1, 1-12 (2019).

35. Tinkle, D. W. The life and demography of the Side-blotched Lizard, Uta stansburiana. Misc. Publ. Museum Zool. Univ. Michigan 1-182 (1967).

36. Zúñiga-Vega, J. J., Fuentes-G., J. A., Ossip-Drahos, A. G. \& Martins, E. P. Repeated evolution of viviparity in phrynosomatid lizards constrained interspecific diversification in some life-history traits. Biol. Lett. 12, 20160653 (2016).

37. Sinervo, B. \& Licht, P. Proximate constraints on the evolution of egg size, number, and total clutch mass in lizards. Science 252, 1300-1302 (1991).

38. Sinervo, B., Doughty, P., Huey, R. B. \& Zamudio, K. Allometric engineering: A causal analysis of natural selection on offspring size. Science 258, 1927-1930 (1992).

39. Méndez-de la Cruz, F. R., Villagrán-Santa Cruz, M. \& Andrews, R. M. Evolution of viviparity in the lizard genus Sceloporus. Herpetologica 54, 521-532 (1998). 
40. Martínez-Méndez, N., Mejía, O., Ortega, J. \& Méndez-de la Cruz, F. R. Climatic niche evolution in the viviparous Sceloporus torquatus group (Squamata: Phrynosomatidae). PeerJ 6, e6192 (2019).

41. Wiens, J. J., Kozak, K. H. \& Silva, N. Diversity and niche evolution along aridity gradient in noth american lizards (Phrynosomatidae). Evolution 67, 1715-1728 (2013).

42. Katoh, K. \& Standley, D. M. MAFFT multiple sequence alignment software version 7: Improvements in performance and usability. Mol. Biol. Evol. 30, 772-780 (2013).

43. Maddison, W. P. \& Maddison, D. R. Mesquite: A modular system for evolutionary analysis. Version 3.61. http://www.mesquiteproject.org (2019).

44. Leaché, A. D., Banbury, B. L., Linkem, C. W. \& Montes-De Oca, A. N. Phylogenomics of a rapid radiation: Is chromosomal evolution linked to increased diversification in north american spiny lizards (Genus Sceloporus)? BMC Evol. Biol. 16, 63 (2016).

45. Yatkola, D. A. Mid-Miocene lizards from western Nebraska. Copeia 1976, 645-654 (1976).

46. Smith, K. T. A diverse new assemblage of Late Eocene squamates (Reptilia) from the Chadron Formation of North Dakota, U.S.A. Palaeontol. Electron. 9, (2006).

47. Bouckaert, R. et al. BEAST 2.5: An advanced software platform for Bayesian evolutionary analysis. PLoS Comput. Biol. 15, e1006650 (2019).

48. Miller, M. A., Pfeiffer, W. \& Schwartz, T. Creating the CIPRES Science Gateway for inference of large phylogenetic trees. In 2010 Gateway Computing Environments Workshop, (GCE) 1-8 (2010).

49. Rambaut, A., Drummond, A. J., Xie, D., Baele, G. \& Suchard, M. A. Posterior 
summarization in Bayesian phylogenetics using Tracer 1.7. Syst. Biol. 67, 901-904 (2018).

50. Jiménez-Arcos, V. H., Sanabria-Urbán, S. \& Cueva del Castillo, R. The interplay between natural and sexual selection in the evolution of sexual size dimorphism in Sceloporus lizards (Squamata: Phrynosomatidae). Ecol. Evol. 7, 905-917 (2017).

51. Meiri, S., Feldman, A., Schwarz, R. \& Shine, R. Viviparity does not affect the numbers and sizes of reptile offspring. J. Anim. Ecol. 89, 360-369 (2020).

52. Benabib, M., Kjer, K. M. \& Sites Jr., J. W. Mitochondrial DNA sequence-based phylogeny and the evolution of viviparity in the Sceloporus scalaris group (Reptilia, Squamata). Evolution 51, 1262-1275 (1997).

53. Angilletta, M. J., Niewiarowski, P. H. \& Navas, C. A. The evolution of thermal physiology in ectotherms. J. Therm. Biol. 27, 249-268 (2002).

54. Hertz, P. E., Huey, R. B. \& Stevenson, R. D. Evaluating temperature regulation by field-active ectotherms: The fallacy of the inappropriate question. Am. Nat. 142, 796-818 (1993).

55. Spellerberg, I. F. Temperature tolerances of Southeast Australian reptiles examined in relation to reptile thermoregulatory behaviour and distribution. Oecologia $\mathbf{9}, 23$ 46 (1972).

56. Bakken, G. S. Measurement and application of operative and standard operative temperatures in ecology. Am. Zool. 32, 194-216 (1992).

57. Fick, S. E. \& Hijmans, R. J. WorldClim 2: new 1-km spatial resolution climate surfaces for global land areas. Int. J. Climatol. 37, 4302-4315 (2017).

58. García-Collazo, R., Altamírano-Álvarez, T. \& Gómez-Soto, M. Reproducción continua en Sceloporus variabilis variabilis (Sauria: Phrynosomatidae) en Alvarado, 
Veracruz, México. Bol. Soc. Herpetol. Mex. 5, 51-59 (1993).

59. Rodríguez-Romero, F. de J. Demografía comparada de dos especies de lacertilios emparentados del genero Sceloporus (Sauria: Phrynosomatidae) con diferente modo reproductor. (Universidad Nacional Autónoma de México, 2004).

60. Ramírez-Bautista, A., Stephenson, B. P., Lozano, A., Uribe-Rodríguez, H. \& Leyte Manrique, A. Atypical reproductive cycles in a population of Sceloporus grammicus (Squamata: Phrynosomatidae) from the Mexican Plateau. Ecol. Evol. 2, 1903-1913 (2012).

61. Jackson, J. F. \& Telford, S. R. Reproductive ecology of the Florida Scrub Lizard, Sceloporus woodi. Copeia 1974, 689-694 (1974).

62. Goldberg, S. R. Reproduction in the Short-Horned Lizard Phrynosoma douglassi in Arizona. Herpetologica 27, 311-314 (1971).

63. Rodríguez-Romero, F., Smith, G. R., Cuellar, O. \& Méndez de la Cruz, F. R. Reproductive traits of a high elevation viviparous lizard Sceloporus bicanthalis (Lacertilia: Phrynosomatidae) from Mexico. J. Herpetol. 38, 438-443 (2004).

64. Rodríguez-Romero, F. et al. Demography of a semelparous, high-elevation population of Sceloporus bicanthalis (Lacertilia: Phrynosomatidae) from the Nevado de Toluca volcano, Mexico. Southwest. Nat. 56, 71-77 (2011).

65. García-Pareja, M., Beltrán-Sánchez, E. \& Sherbrooke, W. C. Phrynosoma asio (Giant Horned Lizard). Natural longevity. Herpetol. Rev. 48, 439-440 (2017).

66. Martínez-Bernal, R. L., Chávez-Tapia, C. B. \& García-Collazo, R. Aspectos de la reproducción, dieta y edad de la especie microendémica Sceloporus macdougalli Smith y Bumzahem, 1953 (Squamata: Phrynosomatidae), de Tehuantepec, Oaxaca. Rev. Zool. 30, 12-35 (2019). 
67. R Core Team. R: A language and environment for statistical computing. R Foundation for Statistical Computing, Vienna, Austria. https://www.r-project.org/ (2020).

68. Huelsenbeck, J. P., Nielsen, R. \& Bollback, J. P. Stochastic mapping of morphological characters. Syst. Biol. 52, 131-158 (2003).

69. Revell, L. J. phytools: An R package for phylogenetic comparative biology (and other things). Methods Ecol. Evol. 3, 217-223 (2012).

70. Beaulieu, J. M., Jhwueng, D. C., Boettiger, C. \& O’Meara, B. C. Modeling stabilizing selection: Expanding the Ornstein-Uhlenbeck model of adaptive evolution. Evolution 66, 2369-2383 (2012).

71. Burnham, K. P., Anderson, D. R. \& Huyvaert, K. P. AIC model selection and multimodel inference in behavioral ecology: Some background, observations, and comparisons. Behav. Ecol. Sociobiol. 65, 23-35 (2011).

72. Pinhero, J., Bates, D., DebRoy, S., Sarkar, D. \& R Core Team, (2020). nlme: Linear and nonlinear mixed effects models. R package version 3.1-11. https://cran.rproject.org/package $=$ nlme.

73. Felsenstein, J., Ackerly, D. D. \& Mcpeek, M. A. A comparative method for both discrete and continuous characters using the threshold model. Am. Nat. 179, 145-156 (2012)

74. Revell, L. J. Ancestral character estimation under the threshold model from quantitative genetics. Evolution 68, 743-759 (2014).

75. Hansen, T. F., Pienaar, J. \& Orzack, S. H. A comparative method for studying adaptation to a randomly evolving environment. Evolution 62, 1965-1977 (2008). 


\section{Acknowledgments}

S.F.D.-G., D.M.A.-M., A.B.-V., F.J.M.-N., and R.S.-B. thanks to Posgrado en Ciencias

Biológicas, Instituto de Biología-UNAM and Consejo Nacional de Ciencia y Tecnología (CONACyT) for the scholarships CVU 478292, 412744, 545201, 775920 and 774550, respectively. This research was supported by projects PAPIIT-UNAM IN210116 and IN212119, and CONACyT PDCPN 2015-1319. We also thank the Dirección General de Vida Silvestre, México for the collecting permits (SGPA/DGVS/01629/16, 01205/17, 02490/17, 06768/17, 002463/18, 002490/18, 002491/18, 003209/18, and 02523/19).

\section{Author contributions}

S.F.D.-G., F.R.M.-C, N.L.M.-M, M.E.O., and M.M.M. design the study. S.F.D.-G., P.G.T., F.R.M.-C, D.M.A.-M., A.B.-M., H.G., R.A.L.-R., C.A.M.-M., F.J.M.-N., R.S.-B., J.H.V.-V., and G.A.W.-P performed field and laboratory work to obtain the physiological, morphological, and life-history data. S.F.D.-G., N.L.M.-M. and A.B.-V built the ultrametric tree. S.F.D.-G. and M.M.M. analyzed the data and drafted the manuscript. All authors contributed to subsequent revisions.

\section{Competing interest}

The authors declare no competing interests. 


\section{Figures}

\section{a. Evolutionary Transitions to Live Birth}
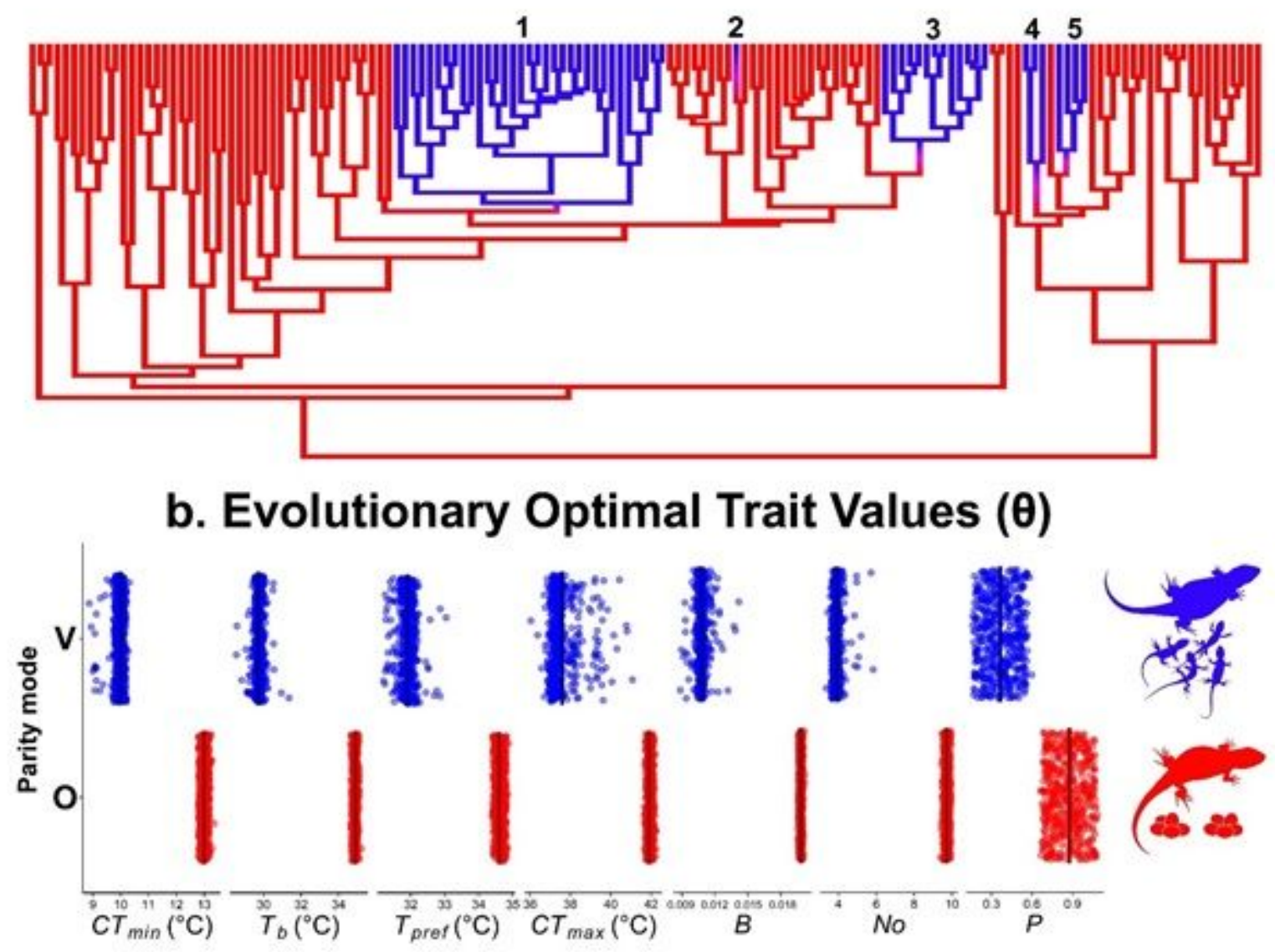

Figure 1

Parallel reductions in physiological and life history traits are associated with viviparity in phrynosomatid lizards. a Five evolutionary transitions from egg-laying (red) to live-bearing (blue) occurred in phrynosomatids. b Viviparous lineages are characterized by reductions in the evolutionary optimal trait values $(\theta)$ for cold tolerance (CTmin), field-estimated body temperature (Tb), the laboratory-measured preferred body temperature (Tpref), heat tolerance (CTmax), mass-specific metabolic rate (B), the annual number of offspring (No), and mass-specific production $(P)$. Evolutionary optimal trait values $(\theta)$ were inferred from the Ornstein-Uhlenbeck (OU) model-fitting procedure (see Methods). Each point represents a different stochastic character map from our analyses ( $n=500$ per trait). 

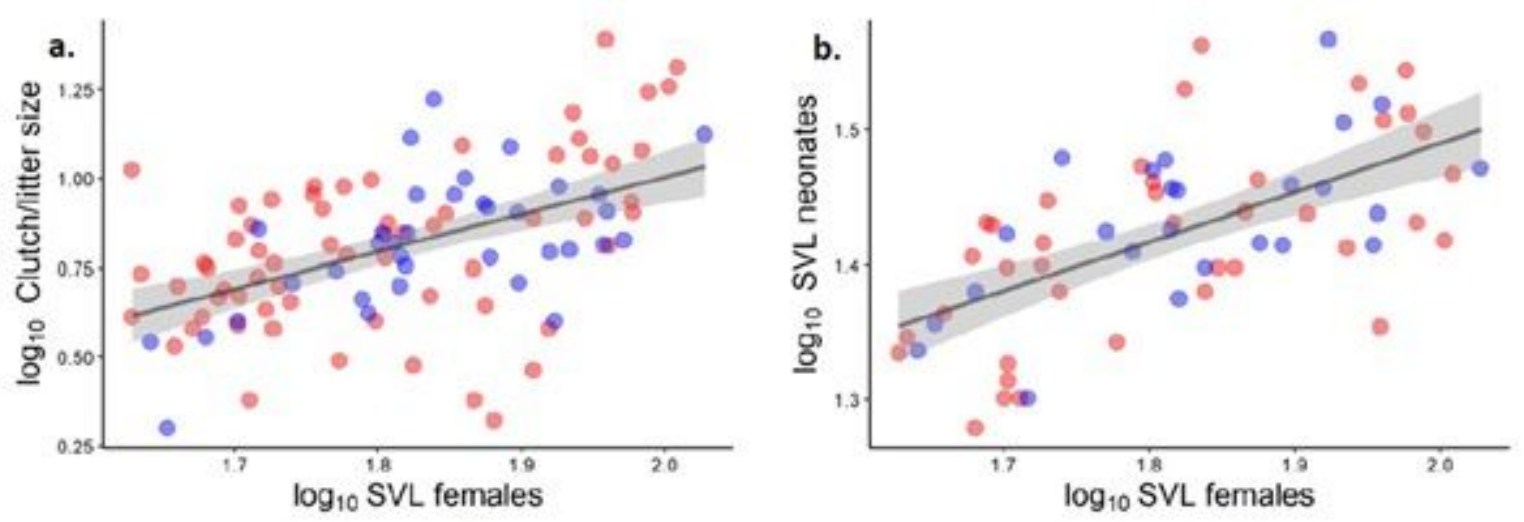

Figure 2

Influence of dam's body size on clutch/litter size ( $a ; y=1.1321 x-1.2362, p<0.01)$ and on the body size of neonates $(b ; y=0.2893 x+0.9064, p<0.01)$ in phrynosomatid lizards. Blue circles correspond to viviparous species whereas red circles correspond to oviparous species. $95 \%$ confidence bands are included around regression lines.
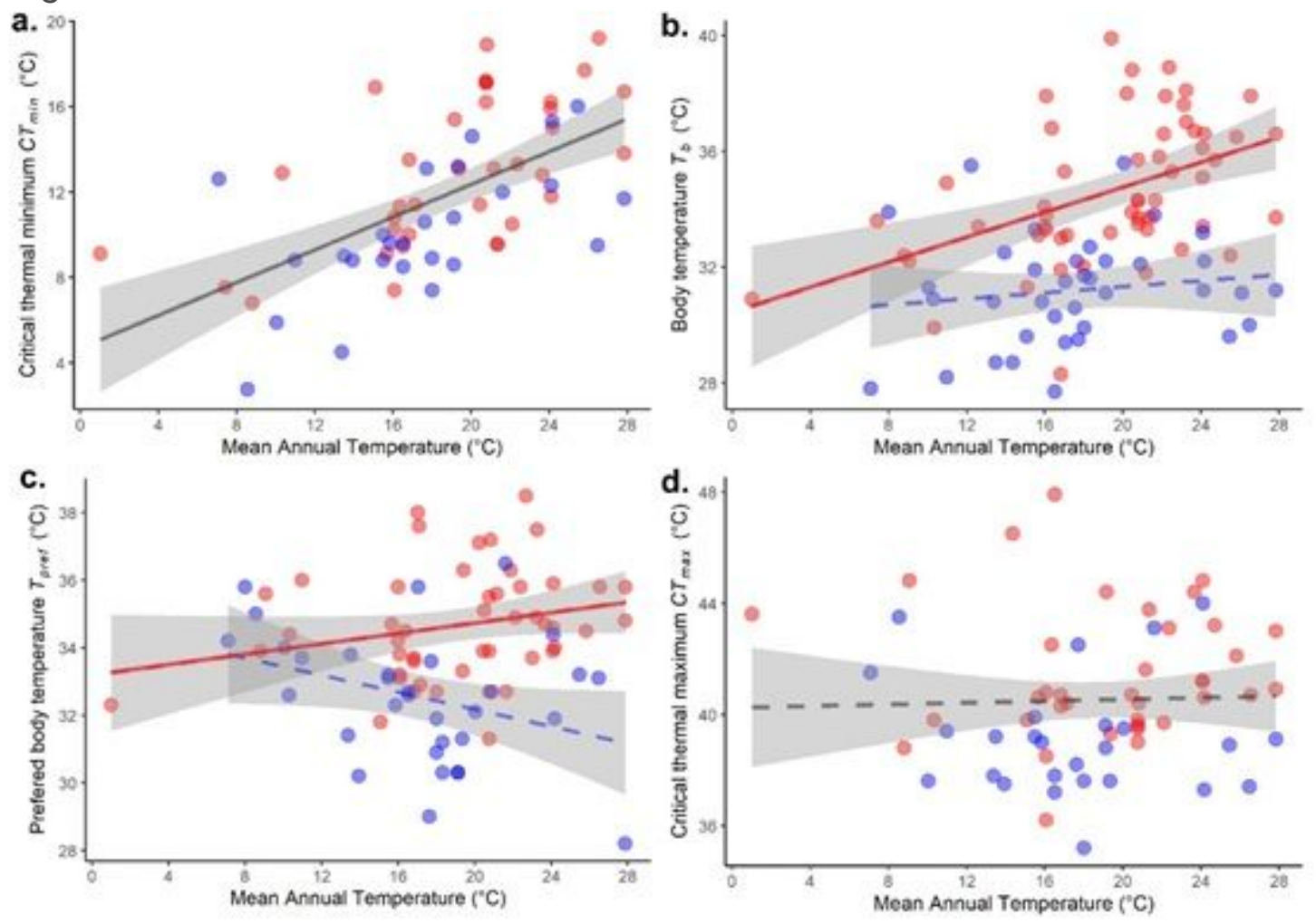

Figure 3

Influence of mean annual temperature (MAT) on cold tolerance $(a ; y=0.354 x+5.639, p=0 ;)$, field-body temperature ( $b$; for oviparous: $y=0.230 x+30.3, p=0$, and for viviparous: $y=0.055 x+30.69, p=0.4$ ), preferred body temperature (c; for oviparous: $y=0.094 x+32.78, p=0.03$, and for viviparous: $y=-0.078 x+$ $34.6, p=0.2)$, and heat tolerance $(d ; y=0.032 x+41.29, p=0.5)$. Blue circles correspond to viviparous species whereas red circles correspond to oviparous species. Solid lines represent slopes with statistical significance $(<0.05)$, and dashed lines represent slopes that are not statistically different from $0.95 \%$ 
confidence bands are included around regression lines. PGLS results using the other macroclimatic predictors (bio10 and bio11) are given in Supplementary Table 4. Parity mode did not impact the relationship between MAT and CTmin (a) or CTmax (d); therefore, data were combined for oviparous and viviparous species (as indicated by a single gray regression line).

\section{Supplementary Files}

This is a list of supplementary files associated with this preprint. Click to download.

- SupplementaryInformation.docx

- SupplementaryData1.xlsx

- SupplementaryData2.xlsx

- PhrynosomatidaeGeneMatrix.txt

- PhrynosomatidaeTree.txt

- Phrynosomatidae500Trees.txt

- RS.pdf 